\title{
Reduced yield stress for zirconium exposed to iodine: reactive force field simulation
}

\author{
Matthew L Rossi ${ }^{1,4^{*}}$, Christopher D Taylor ${ }^{2,4}$ and Adri CT van Duin ${ }^{3}$
}

\author{
* Correspondence: \\ Matthew.Rossi@nrc.gov \\ ${ }^{1}$ Nuclear Regulatory Commission, \\ Rockville, MD 20852, USA \\ ${ }^{4}$ Los Alamos National Laboratory, \\ Los Alamos, NM 87545, USA \\ Full list of author information is \\ available at the end of the article
}

\begin{abstract}
lodine-induced stress-corrosion cracking (ISCC), a known failure mode for nuclear fuel cladding, occurs when iodine generated during the irradiation of a nuclear fuel pellet escapes the pellet through diffusion or thermal cracking and chemically interacts with the inner surface of the clad material, inducing a subsequent effect on the cladding's resistance to mechanical stress. To complement experimental investigations of ISCC, a reactive force field (ReaxFF) compatible with the Zr-I chemical and materials systems has been developed and applied to simulate the impact of iodine exposure on the mechanical strength of the material. We show that the material's resistance to stress (as captured by the yield stress of a high-energy grain boundary) is related to the surface coverage of iodine, with the implication that ISCC is the result of adsorption-enhanced decohesion.
\end{abstract}

Keywords: Zirconium; lodine; Stress corrosion cracking; Failure; Molecular dynamics; Simulation; Reactive force field; Grain boundary

\section{Correspondence/Findings}

Iodine-induced stress-corrosion cracking (ISCC) is a complex phenomenon with significant interest to the nuclear industry. One of the major fission products of uranium is iodine, which is corrosive to zirconium-based cladding. The van-Arkel process [1], developed in 1925, catalogued the reaction between zirconium and iodine for use as a method of metal purification, utilizing the volatility of the tetra-coordinated iodide, $\mathrm{ZrI}_{4}$ [2]. Despite this known reactivity between zirconium and iodine, zirconium was chosen as a cladding material due to its general corrosion resistance, good thermal conductivity, and low cross-section to thermal neutrons [3,4]. There have been a significant number of studies regarding the effects of iodine on $\mathrm{Zr}$-based cladding materials and the pellet-cladding interactions (PCI) which occur on the inner surface of the cladding, such as those by Lyons et al. [5] and Atrens et al. [6]. In work by Lyons et al. [5], and others [7-11], fission products of interest to PCI corrosion have included cesium and cadmium in addition to iodine, all of which have a weakening effect on the mechanical strength of zirconium, as supported in theoretical work by Wimmer et al. [12]. It can be difficult to collect reactor-relevant experimental data due to the irradiation damage, exposure to high-energy atom bombardment, and reactor conditions the cladding is subjected to during its lifecycle. Much of the available experimental data regarding ISCC has been in controlled conditions, utilizing halide-solutions, such as the work of Francon [13], Goryachev [14], and Park [15]. However, it remains 
unknown how the stress-corrosion response initiates $[14,16]$, since post-failure analysis precludes examination of this critical first step.

ISCC can involve multiple, non-linear, and possibly parallel, reaction steps with regards to the overall process. The complex nature of ISCC lies in the governing kinetics and potential pathways of the reaction. The cladding material itself is usually comprised of Zircaloy-4 or Zirlo. The general corrosion process of zirconium alloys has been documented in previous experimental work by Farina [17-19], Francon [13] and Kim [20], among others. Parallel reaction paths can arise due to the iodine decay chain, introducing mechanical effects as iodide phases decompose to chemically inert xenon. Considerations should also be given to the presence of the $8-10 \mu \mathrm{m}$ thick passive oxide layer that protects the inner surface of the cladding [18]. This oxide layer is the first defense of the cladding material from ISCC, and the means via which it is compromised during an ISCC event is unclear [21]. It is possible that synergistic interactions with the electropositive fission products $(\mathrm{Cs}, \mathrm{Cd}, \mathrm{Sr}$, among others) can chemically modify and/or mechanically weaken the oxide, undermining its passivity. The significance of grain-boundaries and triple-points was made apparent in recent investigations by Park [15]. A fundamental approach to modeling ISCC, therefore, should involve a deconstruction of these effects, to assess their individual contributions, prior to a final integration.

In our previous work [22-24], several mechanisms were investigated to help construct such a model. Beginning with the chemical interaction, the series of iodine intermediary states was examined, under the general scheme of iodine aggregation:

$$
\mathrm{Zr}+2 \mathrm{I}_{2} \leftrightarrow \mathrm{ZrI}_{2}+\mathrm{I}_{2} \leftrightarrow \mathrm{ZrI}_{3}+\frac{1}{2} \mathrm{I}_{2} \leftrightarrow \mathrm{ZrI}_{4}
$$

In addition to molecular iodine aggregation, the properties of systems such as surface adsorbates, dimers of molecules, and mixed crystal systems were also modeled as being pertinent to the overall reaction scheme. The focus of the present work is now on the integration of the molecular and crystallographic studies into a molecular dynamics (MD) based model for simulating the interactions between zirconium and iodine. Although the interactions between the oxide and Cs and I, as primary fission products, are also of great interest, the challenges inherent to the development of interatomic potentials for ternary systems and beyond necessitate that we begin with the Zr-I binary. Specifically, we perform molecular dynamic simulations for the interactions of iodine with the 0001 (basal) plane of zirconium, which is known to provide the greatest resistance to ISCC.

While density-functional theory (DFT) was previously utilized to examine the molecular, solid state, and gaseous interactions pertinent to the Zr-I system, [22-24] the approach is computationally expensive and thus impractical for the study of the interaction of I with grain-boundaries that intersect the surface, and its effect on the stressstrain response. ReaxFF, a reactive force field developed by van Duin et al. [25], enables simulation of large systems (i.e. $>10^{4}$ atoms), based on a training set created by molecular and solid-state DFT calculations. ReaxFF MD simulations can predict chemical reactions (including changes in bonding) and diffusion pathways and materials properties while remaining computationally tractable. The specific details of computational models used have been published previously [22-24], and were used in the training set 
for the ReaxFF force field parameters [26]. The Large-scale Atomic/Molecular Massively Parallel Simulator, LAMMPS [27,28], was used to perform the ReaxFF-MD [29] simulations described herein.

Given that ISCC is a joint chemical-mechanical effect, it is critical to explore the role iodine plays in modifying the mechanical response of zirconium metal. Since ISCC primarily occurs in the intergranular mode, we consider the resistance of a tilt grainboundary that intersects the (0001) surface plane to a systematically applied strain rate during molecular dynamics simulation. In order to select the grain boundary most susceptible to this kind of effect, we compare the unrelaxed grain-boundary energies for the slab bicrystals as a function of $\theta$, the angle of rotation about the (0001) axis, Figure 1. Here we compare the grain-boundary energies by scaling the simulations to the equivalent energy per atom (hence units $\mathrm{eV} /$ atom):

$$
\gamma \propto \frac{E_{g b}-E_{g b, \theta=0}}{N_{g b}}
$$

Due to symmetry the energies are periodic every $60^{\circ}$, with reflective symmetry and a local minimum about $30^{\circ}$, at which angle every other plane parallel to the surface is coincident. Based on the relations in Figure 1, we selected a grain boundary with $15^{\circ}$ tilt angle, as this creates a high-energy grain boundary that would be anticipated to be the most vulnerable to reaction with iodine [30]. Since the scope of this study is to demonstrate the utility of Reax-MD simulations to explore the synergies between chemical and mechanical effects, we defer systematic study of other grain boundary systems for future work.

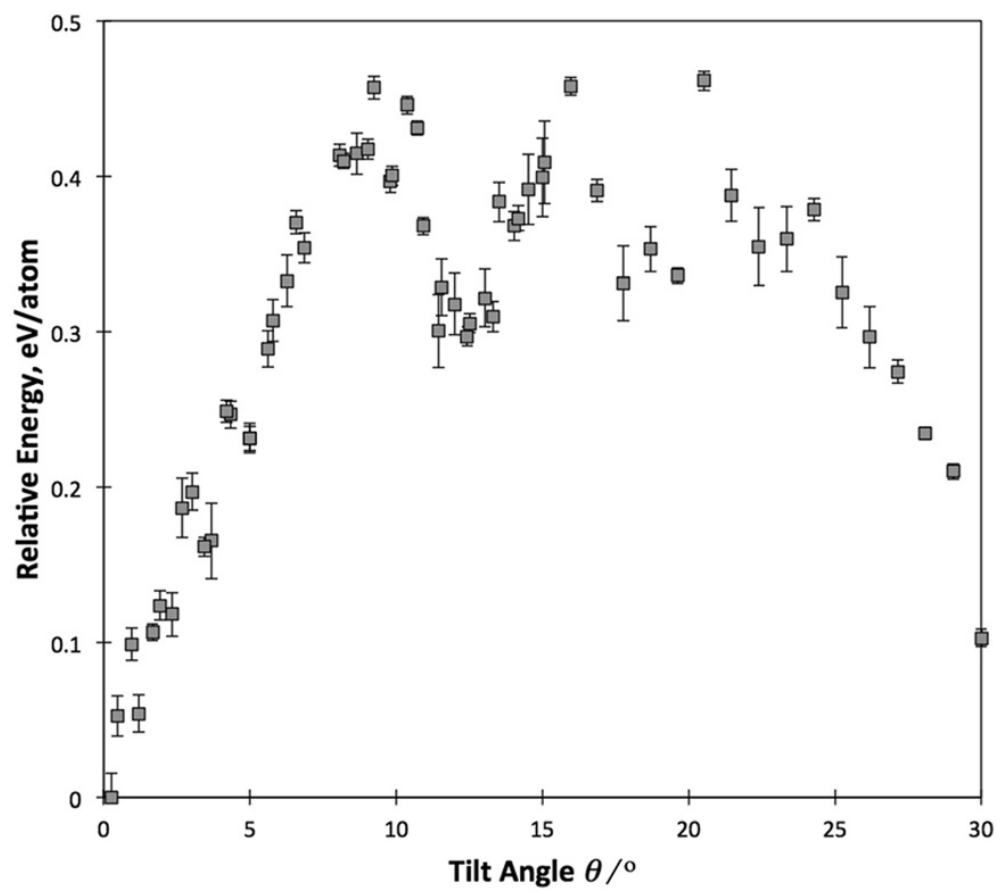

Figure 1 Relative grain boundary energy as a function of tilt angle about the (0001) axis calculated via ReaxFF. The error bars indicate the standard deviation. 
Reax-MD simulations were then performed by introducing varying amounts of iodine into the vacuum space between the periodic slabs of the $\operatorname{Zr}(0001)$-oriented bicrystals. Each simulation contained 31,500 Zr atoms divided into two single crystals 20 atomic layers thick, separated by the grain-boundary. An energy minimization was performed and then the volume and atom positions in the simulation supercell were equilibrated to $500^{\circ} \mathrm{C}$ and $0 \mathrm{MPa}$ in the plane of the bicrystal. Due to the heavy mass of the atoms, a large step size could be used for the MD of $10 \mathrm{fs} /$ timestep. Given the equilibrium cell volume and the number of iodine atoms introduced, the effective partial pressure of iodine was determined. Iodine was introduced in the atomic state, simulating radiolytic release. Iodine may also be present in other chemical states: molecular $\mathrm{I}_{2}$, cesium iodide, or zirconium iodide vapors, but these were not treated within this study. Since it is known that molecular iodine adsorbs onto zirconium metal surfaces without a barrier, it is expected that atomic iodine should behave similarly to molecular iodine [31]. Following this step, MD simulations were performed under conditions of constant strain rate and at a constant temperature of $500^{\circ} \mathrm{C}$. The temperature was selected to be representative of fission gas environments encountered during fuel pellet power cycles. The strain was applied in the direction perpendicular to the grain boundary at a rate of $10^{8} / \mathrm{s}$, or $10 \%$ over the $1 \mathrm{~ns}$ simulation. The stress in this direction was computed and normalized to the slab cross-sectional area to obtain the effective stress corresponding to the strain every 50 time steps. Subsequently, the stress-strain curve for the bicrystal was obtained for a range of iodine partial pressures ranging from 0-12 MPa. Pressures in this range are representative of the fission gas environment within the fuel-pellet gap. Stress-strain data is available directly as an excel file in the Additional file 1.

During our simulations, the maximum yield stress was obtained around 4\% applied strain. The maximum yield stress was captured for each concentration of iodine and the results are plotted in Figure 2 (left axis). The results indicate a rapid fall-off of the grain-boundary resistance to applied stress up to $20 \%$ of the yield stress without iodine, when iodine pressures up to $0.5 \mathrm{MPa}$ are applied. Following this point, the reduction

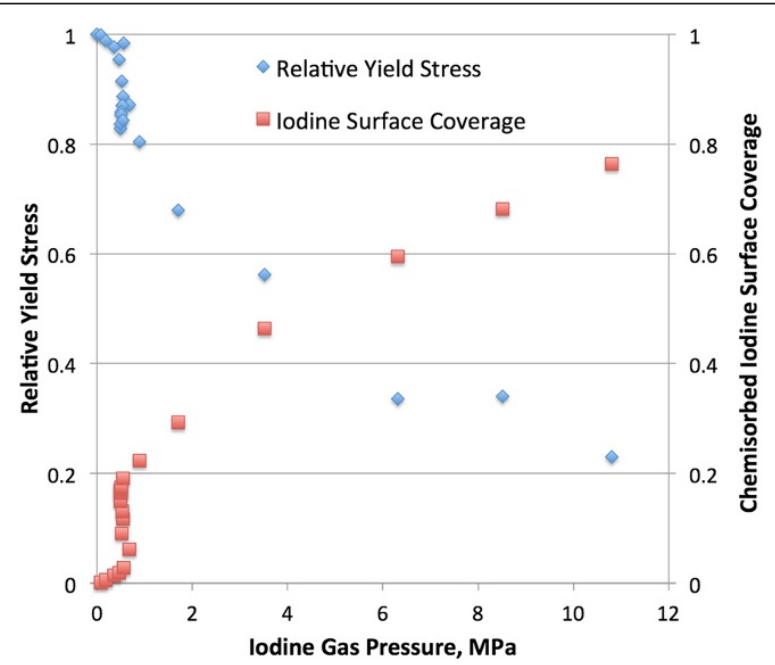

Figure 2 Relative yield stress compared to the iodine-free case (left axis) and the chemisorbed iodine surface coverage (right axis) plotted against the iodine gas pressure (iodine as $I_{2}$ molecules). 
becomes more gradual, with up to $80 \%$ reduction in grain boundary yield stress at $11 \mathrm{MPa}$.

The MD trajectories were then examined to explore the mechanisms for this nonlinear behavior. In the low-pressure region $(<0.5 \mathrm{MPa})$ it was observed that the small number of iodine atoms introduced into the vapor space are almost entirely chemisorbed onto the surface, a process that interferes with the ability to accurately equilibrate the pressure in the gas phase. We use the term chemisorption because it is known that molecular iodine dissociates without a barrier onto the zirconium surface, and so the bonding relation between the undercoordinated $\mathrm{Zr}$ and I is stronger than the covalent $I_{2}$ bond [31]. As seen in Figure 2 (right axis) the surface chemisorption follows a similar non-linear response as a function of pressure, indicating that there is a direct connection between the surface chemisorption phenomena and the reduction in the applied stress. We, therefore, chose to discard pressure as a significant variable for pressures $<0.5 \mathrm{MPa}$ and evaluated the relative yield stress as a function of chemisorbed iodine surface coverage. When the data is presented in this way (Figure 3) the relation becomes strongly linear, indicating that the extent to which the monolayer iodide film is allowed to form has a significant impact on the stress required to initiate materials failure at the point of the grain-boundary.

Examination of the molecular dynamics trajectory about the yield point (Figure 4) indicates that yielding is initiated by the creation of excess surface area at the point where the grain boundary intersects the surface (i.e. crack initiation). In both of the cases shown in Figure 4, the formation of that yield point is accompanied by ingress of iodine

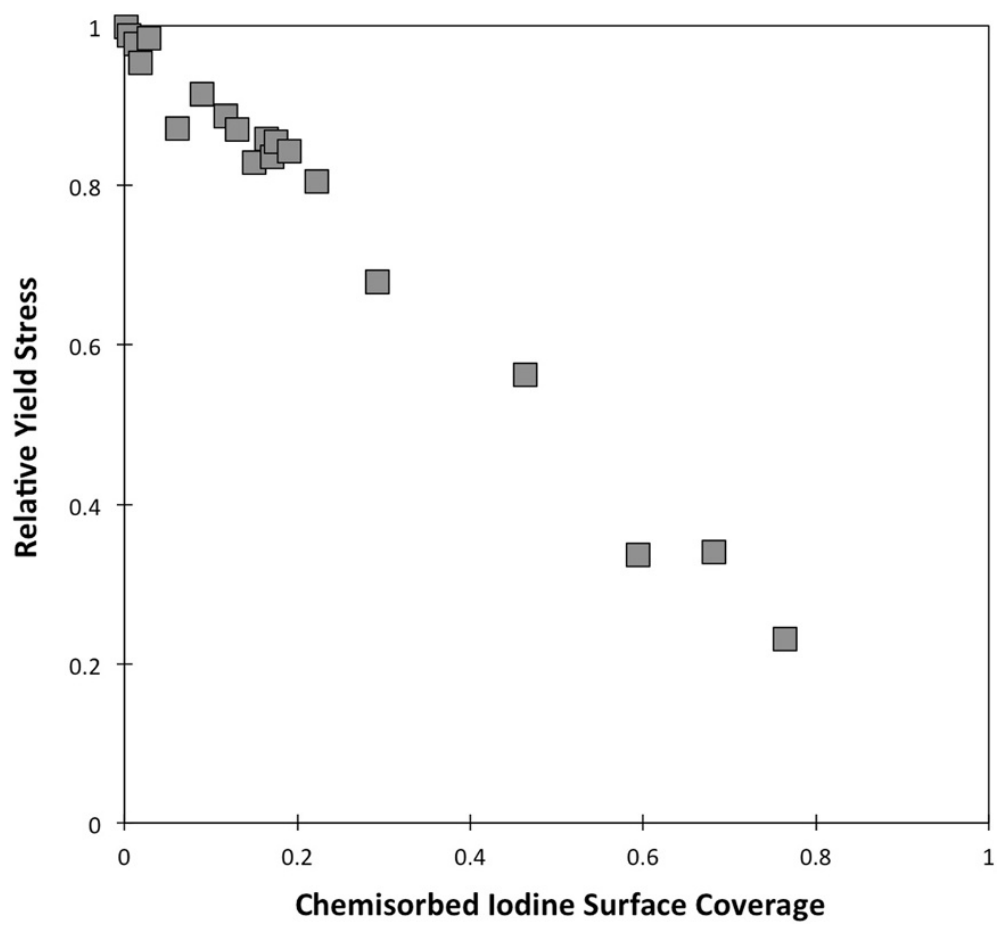

Figure 3 The relative maximum yield stress obtained from the constant strain rate molecular dynamics simulation plotted against the chemisorbed iodine surface coverage, calculated as the number of chemisorbed iodine atoms per surface zirconium atoms. 


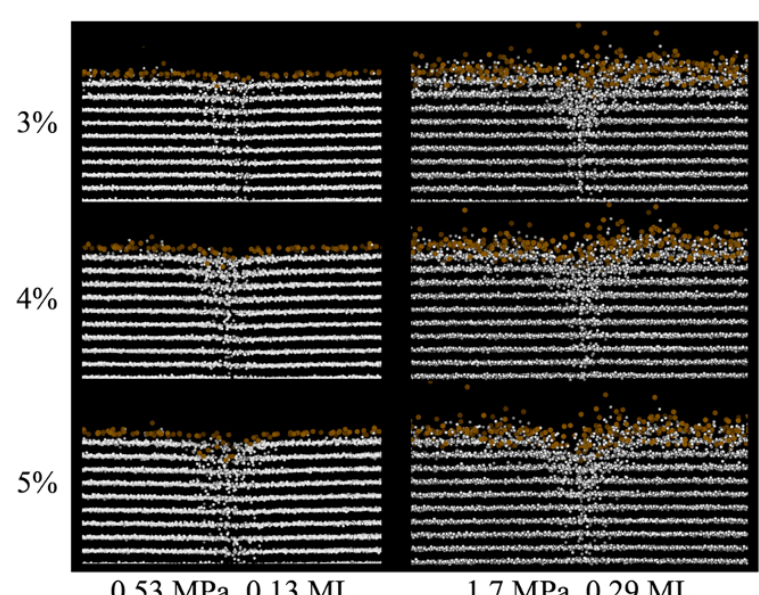

Figure 4 Snapshots taken from the molecular dynamics simulation at three different values of strain that span the yield point, which occurs at around $4.5 \%$ strain. The snapshots are shown for $0.53 \mathrm{MPa}$ and $1.7 \mathrm{MPa}$ iodine exposure.

into the crack region. The ability for iodine to migrate rapidly across the surface, [31] in addition to its propensity to form multicoordinate bonds to the freshly exposed $\mathrm{Zr}$ atoms [22] lowers the surface energy, and, consequently, the energy penalty associated with the yield phenomenon. In essence, this is similar to the enhanced decohesion mechanism of hydrogen-induced cracking [32].

A further distinction noted in Figure 4 relates to the change in iodine film structure between $0.53 \mathrm{MPa}$ and 1.7 MPa: In the lower pressure case, the iodide film constitutes a partial monolayer, at the higher pressure, a phase transformation has occurred in the surface phase, resulting in the intermingling of surface $\mathrm{Zr}$ and I atoms, according to the agglomeration pathway outlined above [2]. The reorganization of the surface layers that accompanies the surface transformation provides an additional impetus for initiating the crack-opening at the grain-boundary/surface intersection, although the mechanistic aspects associated with this should be explored more thoroughly in future work. While very little direct incorporation of iodine into the grain boundary was observed, it has been speculated that grain boundaries facilitate diffusion of iodine into the material due to their excess volume. Relative to the chemisorption effect, however, this mechanism is slow and the material weakening observed herein is a consequence of the iodide film formation and subsequent disorder that it introduces into the surface layers of the material, which result in overall weakening and enhanced susceptibility to mechanical deformation.

In summary, the reactive force field simulations demonstrate that iodine chemisorption leads to a reduction in the resistance of a high-energy grain-boundary in zirconium metal to applied stress, and undergoes a chemisorption mediated reaction to form a zirconium iodide film. The extent to which the relative yield stress is lowered is linearly related to the surface coverage of adsorbed iodine. The methodology demonstrated in this work can now be extended to explore the mechanistic interactions between iodine, iodide-films and stress states at the intersection between the grain-boundary and the surface, as well as the volatilization of zirconium iodide under reactor conditions. 


\section{Additional file}

Additional file 1: Stress-strain data and relevant computations based on the raw data and the stress-strain figures are presented in this additional excel file.

\section{Abbreviations}

DFT: Density functional theory; ISCC: Iodine-induced Stress Corrosion Cracking; LAMMPS: Large-scale Atomic/Molecular Massively Parallel Simulator; MD: Molecular Dynamics; PCI: Pellet-clad interaction; ReaxFF: Reactive Force Field.

\section{Competing interests}

The authors declare that they have no competing interests. This journal article was prepared, in part, by an employee of the U.S. Nuclear Regulatory Commission on his own time apart from his regular duties. NRC has neither approved nor disapproved its technical content.

\section{Authors' contributions}

MR developed the ReaxFF potential and performed DFT and LAMMPS simulations along with data analysis. CT also performed LAMMPS simulations and analysed the results. AvD provided assistance with development of the ReaxFF potential. All authors played contributing roles in the writing and approval of this final manuscript.

\section{Acknowledgment}

This research was performed at Los Alamos National Laboratory and was supported by the Consortium for Advanced Simulation of Light Water Reactors (www.casl.gov), an Energy Innovation Hub (http://www.energy.gov/hubs) for Modeling and Simulation of Nuclear Reactors under U.S. Department of Energy Contract No. DE-AC05-00OR22725. The Los Alamos National Security LLC for the National Nuclear Security Administration of the U.S. Department of Energy under contract DE-AC52-06NA25396. The authors would like to acknowledge Robert Montgomery (PNNL) for his valuable input.

\section{Author details}

${ }^{1}$ Nuclear Regulatory Commission, Rockville, MD 20852, USA. Fontana Corrosion Center, The Ohio State University, Columbus, OH 43210, USA. ${ }^{3}$ Mechanical and Nuclear Engineering, Pennsylvania State University, University Park, PA 16802, USA. ${ }^{4}$ Los Alamos National Laboratory, Los Alamos, NM 87545, USA.

Received: 3 July 2014 Accepted: 22 October 2014

Published online: 04 November 2014

\section{References}

1. van Arkel AE, de Boer JH (1925) Darstellung von reinem Titanium-, Zirkonium-, Hafnium- und Thoriummetall. ZAAC 148(1):345-350

2. Cubicciotti D, Lau KH, Ferrante MJ (1978) Thermodynamics of vaporization and high temperature enthalpy of zirconium tetraiodide. J Electrochem Soc 125:972

3. (1994) Concise Encyclopedia Chemistry. Walter De Gruyter Inc, trans: Eagleson M

4. Fairchild HB (1949) The Properties of Zirconium and Its Possibilities for Thermal Reactors. Laboratory, Oak Ridge National Laboratory, Oak Ridge, TN

5. Lyons MF, Coplin DH, Jones GG (1963/1964) High Performance UO 2 Fuel Program. Quarterly Progress Reports. General Electric Company, GEAP-3771-15

6. Atrens A, Dannhäuser G, Bäro G (1984) Stress-corrosion-cracking of Zircaloy-4 cladding tubes. J Nuc Mater 126:91-102

7. Cox B, Surette BA, Wood JC (1981) In: McNitt RP, Sisson RD (eds) Environmental Degradation of Engineering Materials in Aggressive Environments. Pennsylvania State University, State College, PA, p 293

8. Cox B (1990) Pellet-Clad Interaction (PCI) Failures of Zirconium Alloy Fuel Cladding - A Review. J Nuc Mater 172:249-292

9. Götzmann O (1982) Thermochemical Evaluation of PCI Failures in LWR Fuel Pins. J Nuc Mater 107:185-195

10. Konashi K, Kamimura K, Yokouchi Y (1984) Estimation of Irradiation Induced lodine Pressure in an LWR Fuel Rod. J Nuc Mater 125:244-247

11. Grimes RW, Ball RGJ, Catlow CRA (1992) Site preference and binding of iodine and caesium in uranium dioxide. J Phys Chem Solids 53(4):475-484

12. Wimmer E, Najafabadi R, Young GA Jr, Ballard JD, Angeliu TM, Vollmer J, Chambers JJ, Niimi H, Shaw JB, Freeman C, Christensen M, Wolf W, Saxe P (2010) Ab initio calculations for industrial materials engineering: successes and challenges. J Phys Condens Matter 22:384215

13. Francon V, Fregonese M, Abe H, Watanabe Y (2012) lodine-Induced Stress Corrosion Cracking of Zircaloy-4: Identification of Critical Parameters Involved in Intergranular to Transgranular Crack Propagation. Solid State Phenomena 183:49-56

14. Goryachev SB, Gritsuk AR, Prasolov FF, Snegirev MG, Shestak VE (1992) lodine induced SCC of Zr alloys at constant strain rate. J Nuc Mater 199:50-60

15. Park SY, Kim JH, Choi BK, Jeong YH (2007) Crack initiation and propagation behavior of zirconium cladding under an environment of iodine-induced stress corrosion. Metals and Mat Intl 13(2):155-163

16. Likhanskii W, Matweev LV (2002) The development of the crack growth model in zirconium claddings in iodine environment. Nuc Eng Des 213:133-140

17. Farina SB, Duffo GS, Galvele JR (2002) Localized corrosion of zirconium and zircaloy-4 in iodine alcoholic solutions. LAAR 32:295-298 
18. Farina SB, Duffo GS, Galvele JR (2002) Stress Corrosion Cracking of Zircaloy-4 in Halide Solutions. Effect of Temperature. Mat Res 5:107-112

19. Farina SB, Duffo GS (2004) Intergranular to transgranular transition in the stress corrosion cracking of Zircaloy-4. Corr Sci 46:2255-2264

20. Kim H-G, Jeong Y-H (2006) Effect of Annealing Conditions on the Microstructure and Corrosion Characteristics of Zr-xNb Alloys. Solid State Phenomena 118:77-82

21. Lewis BJ, Thompson WT, Kleczek MR, Shaheen K, Juhas M, Iglesias FC (2011) Modelling of iodine-induced stress corrosion cracking in CANDU fuel. Journal of Nuclear Materials 408(3):209-223, doi:10.1016/j.jnucmat.2010.10.063

22. Rossi ML, Taylor CD (2011) Atomistic Simulations of Formation of Elementary Zr-I Systems. O J Phys Chem 1:104-108

23. Rossi ML, Taylor CD (2013) Equations of State for Crystalline Zirconium lodide: The Role of Dispersion. J Nuc Mater 433(1-3):30-36

24. Rossi ML, Taylor CD (2013) First-principles Insights into the Nature of Zirconium-lodine Interactions and the Initiation of lodine-induced Stress-Corrosion Cracking [in revision]. J Nuc Mater

25. van Duin ACT, Dasgupta S, Lorant F, Goddard WA 3rd (2001) ReaxFF: A reactive force field for hydrocarbons, J Phys Chem A 105:9396-9409

26. ReaxFF potentials available upon request. Contact acv13@psu.edu.

27. Large-scale Atomic/Molecular Massively Parallel Simulator. http://lammps.sandia.gov. Accessed August 62012

28. Plimpton SJ (1995) Fast Parallel Algorithms for Short-Range Molecular Dynamics. J Comp Phys 117:1-19

29. Chenoweth K, van Duin ACT, Goddard WA III (2008) ReaxFF Reactive Force Field for Molecular Dynamics Simulations of Hydrocarbon Oxidation. J Phys Chem A 112(5):1040-1053

30. Musienko A, Cailletaud G (2009) Simulation of inter- and transgranular crack propagation in polycrystalline aggregates due to stress corrosion cracking. Acta Mater 57:3840-3855

31. Legris A, Domain C (2005) Ab initio atomic-scale modeling of iodine effects on hcp zirconium. Phil Mag 85:589-595

32. Van der Ven A, Ceder G (2002) The thermodynamics of decohesion. Acta Mat 52:1223-1235

doi:10.1186/s40323-014-0019-z

Cite this article as: Rossi et al:: Reduced yield stress for zirconium exposed to iodine: reactive force field simulation. Advanced Modeling and Simulation in Engineering Sciences 2014 2:19.

Submit your manuscript to a SpringerOpen ${ }^{\circ}$ journal and benefit from:

- Convenient online submission

- Rigorous peer review

- Immediate publication on acceptance

- Open access: articles freely available online

- High visibility within the field

- Retaining the copyright to your article

Submit your next manuscript at $>$ springeropen.com 